\title{
What does the GB power outage on 9 August 2019 tell us about the current state of decarbonised power systems?
}

\author{
Janusz Bialek \\ Newcastle University, Merz Court, Newcastle upon Tyne, NE1 7RU, United Kingdom \\ Skolkovo Institute of Science and Technology (Skoltech), 121205 Moscow, Bolshoy \\ Boulevard 30, bld. 1, Russian Federation \\ Email: Janusz.Bialek@ncl.ac.uk
}

\section{Abstract}

The GB power blackout, that happened on 9 August 2019, was a unique stress test exposing fault lines brought about by the rapid changes due to the decarbonisation drive and penetration of smart grids technologies. It has demonstrated that, as a significant amount of new equipment and controls were added to the system in a very short time, the probability of common, hidden modes of failures has significantly increased. In the face of declining reliability, maintaining the status quo is not an option. While currently increasing the $(\mathrm{N}-1)$ security margin could prove to be expensive, the balance of costs and benefits is likely to change in future. Especially wider application of innovative frequency controls, including "virtual inertia" and Remedial Action Schemes, could help reduce the costs. Distributed Generation (DG) reached such a high penetration level that it cannot be treated any longer as negative demand. Traditional underfrequency load shedding should be made more selective. Interactions between the power system and other infrastructures are still poorly understood and there is a significant risk that if the current compartmentalised approach to their governance and operation is not changed, 
we may see more unexpected consequences of disturbances across the whole system.

Keywords: power blackouts, decarbonisation, reliability standards

\section{INTRODUCTION}

Over the last 10-15 years, power systems in many countries have changed quite rapidly and significantly with renewables replacing traditional generation, increasing penetration of distributed generation (DG) in distribution networks, and increasing deployment of energy storage, active demand and smart grids technologies. Hence it is of utmost importance to understand how those changes influence not only the security of supply but also the operation of other linked infrastructures such as e.g. transport. A unique opportunity to help to answer that question was a stress test provided by the $\mathrm{GB}^{1}$ power outage on $9^{\text {th }}$ August 2019 that affected over 1 million customers and caused a major disruption to rail services. Any weaknesses exposed by the outage have to be urgently addressed as the pace of changes will increase due to the UK adopting the target of net-zero greenhouse gases emissions by 2050 . While this paper concentrates on GB, the findings are general and should be of interest to other countries undergoing similar changes.

We start with Section 2, providing an overview of the mechanisms by which power systems are kept secure in the face of generation outages. After describing the event itself in Section 3, we consider the implications in Section 4. We argue that business-as-usual is not sustainable due to reduced observability and an increased probability of unexpected common-mode

\footnotetext{
${ }^{1}$ Great Britain (GB) power system is a part of the United Kingdom (UK) and contains synchronously connected networks of England, Wales and Scotland - but not Northern Ireland which is operated jointly with the Republic of Ireland.
} 
failures. A cost-benefit analysis shows that increasing the (N-1) security margin could prove to be expensive now. However a wider application of innovative frequency controls, which we discuss in some detail, will help to reduce the costs. We also discuss the need to understand better interactions of the power system with other infrastructures. We conclude and discuss policy implications in section 5 .

\section{HOW TO MAINTAIN A RELIABLE SUPPLY OF ELECTRICITY IN PRESENCE OF GENERATION FAILURES}

In this section, we will outline the main principles of maintaining the reliability of a power system and means of achieving it. We will concentrate on keeping the balance of power, and we will not consider the effects of transmission network failures.

\subsection{Power-frequency mechanism and the role of inertia}

Due to a lack of large-scale energy storage, the amount of power produced must be equal to the amount of power consumed. If there is any imbalance, it will show itself by changes in power system frequency which is determined by the speed of synchronous generators which convert mechanical power providing by turbines (steam, gas, hydro) into electrical power in traditional power stations. Synchronous generators are kept in synchronism by electromagnetic forces, i.e. they rotate at the same electrical speed, which means that the frequency is the same everywhere within an $\mathrm{AC}$ interconnection. If there is a disturbance (like tripping of a power station), the balance of forces is disturbed and frequency will change ${ }^{2}$. The inertia of all the rotating elements (generator rotors and turbines) stores kinetic energy, so it provides a buffer

\footnotetext{
${ }^{2}$ Frequency is the same everywhere only on average. The electromagnetic forces exhibit a spring-like behaviour. Hence, if there is a disturbance, the frequency of individual generators will oscillate around the average frequency.
} 
to any power balance disturbances. It will release energy by slowing down of all generators (so frequency will drop) when there is a deficit of power or it will store excess energy by speeding up (so frequency will rise) when there is a surplus of power. Frequency is therefore an indicator of power imbalance. As the balance of generation and demand changes all the time, frequency undergoes continuous fluctuations around the nominal value (50 Hz in GB). The bigger the system inertia, the lower frequency changes resulting from power balance disturbances.

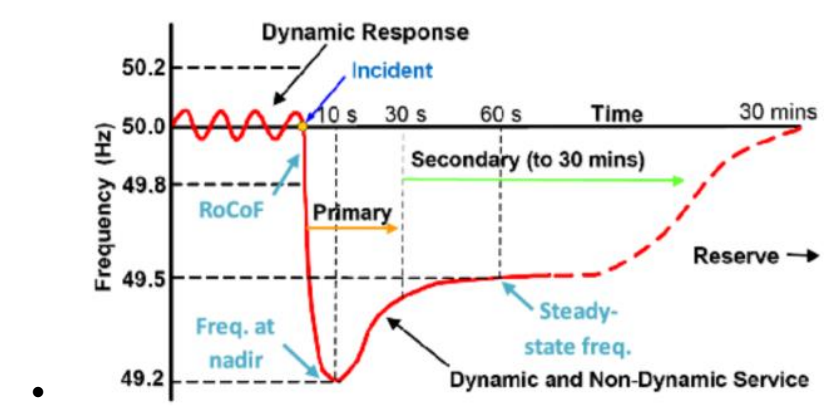

- Figure 1 Illustration of frequency response (Teng, 2015).

Let us now consider what happens when a power station suddenly trips and frequency starts to fall. Figure 1 shows a typical trace of the frequency, also illustrating specific terms used in the paper. The initial speed with which frequency falls is referred to as Rate of Change of Frequency $(\mathrm{RoCoF})$ and it is proportional to the size of the disturbance $\Delta \mathrm{P}$ and inversely proportional to the inertia constant $\mathrm{H}$ :

$$
\operatorname{RoCoF} \propto(\Delta \mathrm{P} / \mathrm{H})
$$

Initially the power imbalance is covered entirely from the kinetic energy stored in system inertia and it is proportional to $\mathrm{RoCoF}-$ see (1). The fall in frequency is sensed by turbine governors so that, after a few seconds delay ${ }^{3}$, the turbines start to react increasing their power

\footnotetext{
${ }^{3}$ The turbine governor and the turbine itself can be modelled by first or higher-order order lag systems which
} 
output. Usually droop control is executed, i.e. the power increase is proportional to the frequency deviation $\Delta \mathrm{f}$ from the nominal frequency. This reduces the slope of frequency changes $\mathrm{df} / \mathrm{dt}^{4}$ and continues until eventually the turbines cover the whole power deficit $\Delta \mathrm{P}$, and frequency stabilises at a steady-state value after a dip called the frequency nadir. Generally, the bigger system inertia $\mathrm{H}$, the smaller RoCoF and frequency nadir. On the other hand, the stronger droop control of the turbines, the lower deviation of the steady-state frequency from $50 \mathrm{~Hz}$. The control process is referred to as primary frequency response - it is fully automatic and all generators synchronised to the grid must participate in it.

The steady-state frequency is less than $50 \mathrm{~Hz}$ as some energy stored in inertia has been taken off to cover the initial power deficit. The task of returning to $50 \mathrm{~Hz}$ is referred to as the secondary frequency response and it is initiated and controlled centrally by SO. It is much slower than the primary frequency control. Usually, only selected generators contribute to it. In order to maintain system stability, System Operator has to keep an appropriate amount of fast-responding primary frequency reserve and slower secondary frequency reserve.

It is essential to consider the impact of renewable generators on the system frequency response. Generally, wind and solar PV power stations do not participate directly in frequency control as they could provide only regulation down but not up, unless they operate part-loaded. Also, they do not contribute to the system inertia as they are connected to the system by power electronics devices, called converters ${ }^{5}$, rather than synchronous machines. Consequently, the system inertia has been dropping for years in GB and other countries as the penetration of wind and

means that they cannot react instantaneously to the changes in in frequency.

${ }^{4} \mathrm{RoCoF}$ is the initial rate-of-change-of-frequency after a disturbance while the slope $\mathrm{df} / \mathrm{dt}$ changes all the time. RoCoF is equal to $\mathrm{df} / \mathrm{dt}$ only initially, before the droop control of turbines is activated.

${ }^{5}$ A converter is a power electronics device that converts AC electricity into DC or DC into AC. 
solar power increases. Reduced inertia results in a bigger RoCoF and frequency nadir and therefore can have serious consequences for system security. This issue is further discussed in Section 4.

\subsection{System Security and Quality of Supply Standard (SQSS)}

In GB, reliability standards are formalised in National Electricity Transmission System Security and Quality of Supply Standard (SQSS, 2019) and the task to enforce them falls to the Electricity System Operator (ESO). To simplify the industry jargon, the system should remain secure following any (N-1) event, i.e. a single infeed loss, referred to as the Secured Event. On 9 August, the largest infeed was an interconnector at 1,000 MW, so the amount of the primary frequency reserve held was $1022 \mathrm{MW}$, while the amount of the secondary reserve was 1314 MW (NGESOa, 2019).

The SQSS anticipates that only one Secured Event would happen at any one time and does not assume multiple Secured Events occurring simultaneously, i.e. (N-2) or more events. The (N1) standard is a common-sense engineering rule, which has been widely accepted around the world. It follows from a consideration that the probability of two power stations failing independently and at the same time is very low and securing against it would be prohibitively expensive.

\subsection{Loss of Mains protection}

When identifying the largest infeed loss, the ESO must also take into account the effect of any consequent infeed losses of Distributed Generators (DG) connected to the distribution network. The reason for that is that any DG must be equipped with Loss of Mains protection which 
disconnects the plant in the event of a loss of supply to the distribution network (islanding) ${ }^{6}$. However, large disturbances at the transmission level could be wrongly interpreted as Loss of Mains by the protection of DG, which would disconnect them. Hence the ESO must make sure that a Secured Event would not activate Loss of Mains protection.

There are two main types of Loss of Mains protection: Rate of Change of Frequency (RoCoF) and Vector Shift. RoCoF protection works on the principle that if a part of a distribution network is islanded, the frequency will change rapidly due to low inertia of that part of the system and a significant imbalance of power ${ }^{7}$. Currently the ESO assumes that the RoCoF limit of DG is set at a historical value of $0.125 \mathrm{~Hz} / \mathrm{s}$ and seeks to ensure that the limit is not breached for any Secured Event. This is achieved by dispatching traditional generation (increasing inertia), management of response and reduction in the size of the potentially largest infeed loss. There is an ongoing Accelerated Loss of Mains Change Programme, due to be completed in 2022, to replace the protection settings of embedded generation to make them less sensitive to transmission system disturbances, and increase RoCoF to $1 \mathrm{~Hz} / \mathrm{s}$.

Vector Shift protection detects sudden changes in the mains voltage angle which often occur during islanding. However, Vector Shift protection has also been found sensitive to shortcircuits at the transmission level, such as those accompanying lightning strikes.

\subsection{Load shedding}

If frequency keeps falling despite activating frequency response, automatic under-frequency load shedding is activated to disconnect demand and restore the power balance. In GB, this is

\footnotetext{
${ }^{6}$ In some countries, distribution networks can form microgrids in which DGs keep generating to continue to supply customers in the event of islanding, but in GB this is not practised.

${ }^{7}$ RoCoF-based Loss of Mains protection is used in GB and Ireland but not necessarily in continental Europe.
} 
referred to as Low Frequency Demand Disconnection (LFDD) and is activated in blocks with the first block activated when the frequency drops to $48.8 \mathrm{~Hz}$. Then $5 \%$ of demand, based on the annual peak demand, is supposed to be disconnected in England and Wales only.

It is essential to appreciate that load shedding is pre-planned and executed by Distribution Network Operators (DNOs) by opening circuit breakers at $33 \mathrm{kV}$ level and therefore disconnecting indiscriminately all the customers connected to the disconnected feeders. This may also include DG and frequency response units hence weakening the effectiveness of load shedding, as indeed happened on 9 August.

\section{DESCRIPTION OF EVENTS}

The description of events is based on (NGESO, 2019b), (Ofgem, 2020) and (E3C, 2020). Before the initial fault, the demand was about $29 \mathrm{GW}$ with about $2 \mathrm{GW}$ provided by solar and $12 \mathrm{GW}$ provided by wind generation ( $2 \mathrm{GW}$ embedded and $10 \mathrm{GW}$ transmission-connected). Generation margins for the day were comfortable. Weather conditions on the day were not unusual, with several yellow warnings of high winds and lightning strikes alerts issued by the Met Office.

\subsection{First Stage: first $\mathbf{4 5}$ secs after the lightning strike}

At 16:52:33 there was a lightning strike on the Eaton Socon - Wymondley 400kV line - see a map of the area in Figure 2. A lightning strike is nothing unusual, and the protection systems on the transmission system operated correctly to clear it. A lightning strike is effectively a short-circuit causing the voltage to drop. The associated voltage disturbance was in line with what was expected and should not have caused any significant disturbances. However, the lightning strike did cause three types of infeed losses totalling $1480 \mathrm{MW}$ discussed in detail below. Figure 3 shows the resulting frequency trace divided into three stages. 


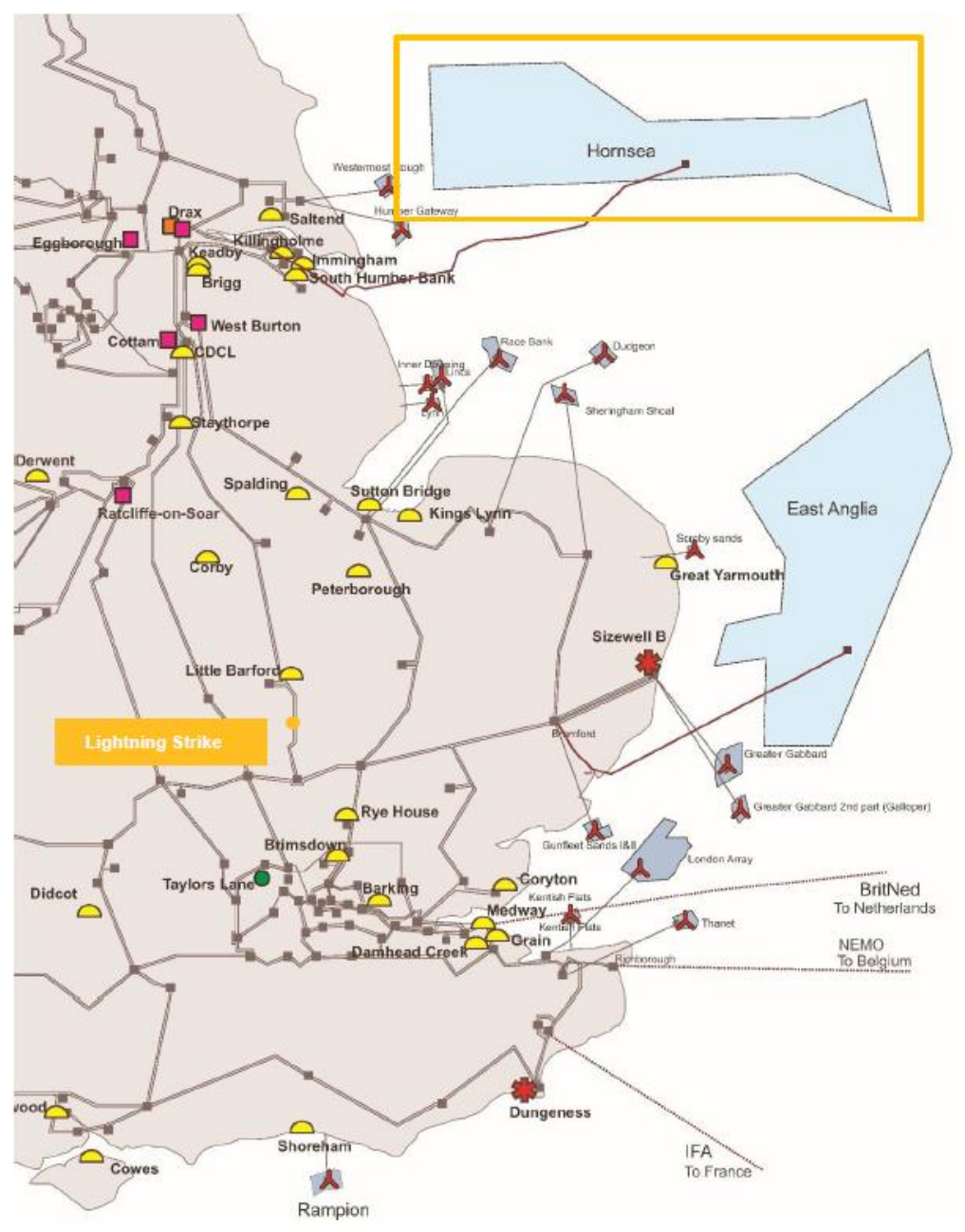

Figure 2 - Map of the affected area (NGESO, 2019) 


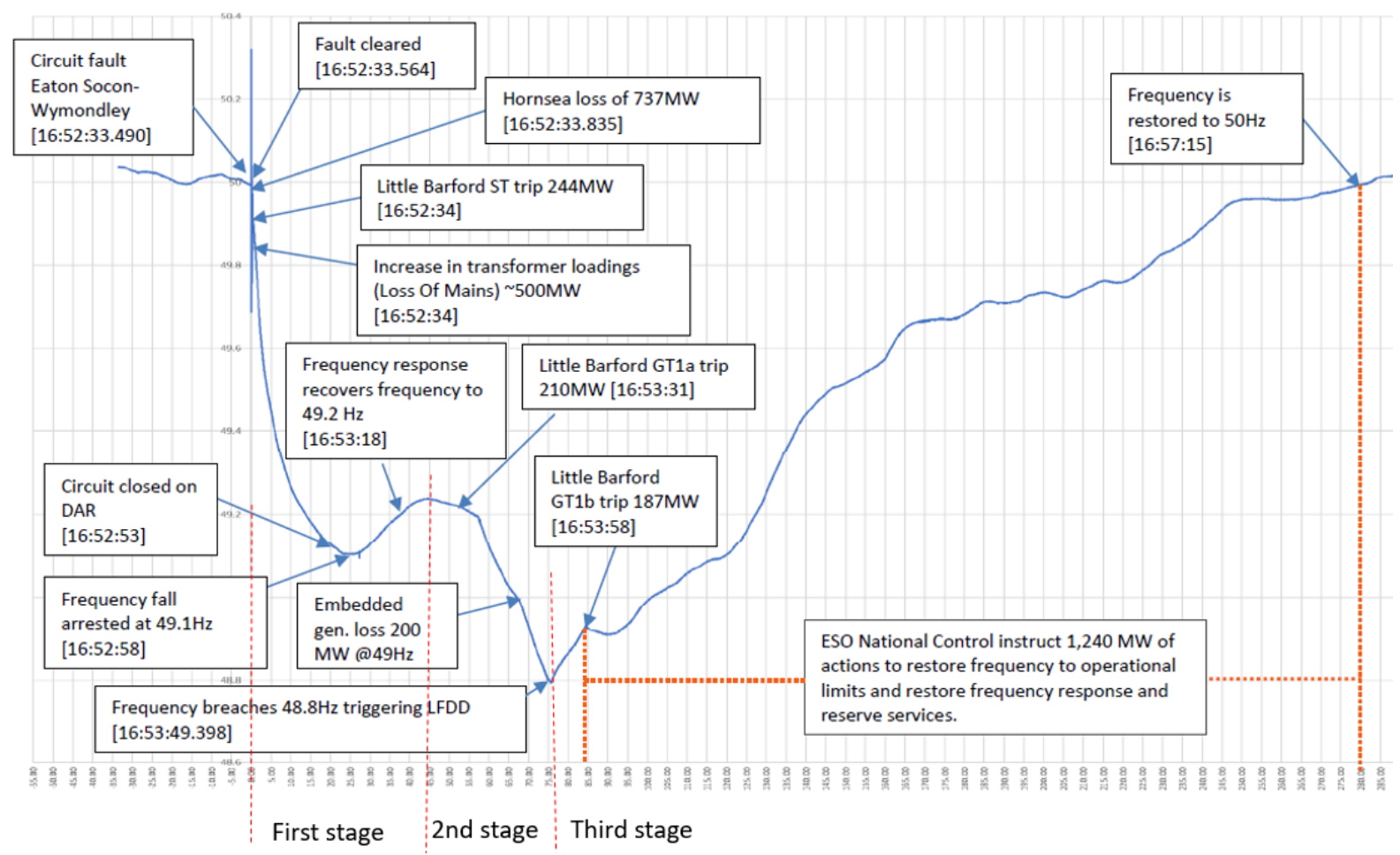

Figure 3: Annotated frequency trace (NGESO, 2019).

\subsubsection{Loss of 737 MW at Hornsea off-shore wind farm}

Hornsea is owned by Ørsted and was connected to the grid in February 2019, i.e. 7 months before the outage. At the time, it was progressing through the Grid Compliance process and had fulfilled the requirements to export on an interim basis only. Shutting down of two units generating $737 \mathrm{MW}$ was triggered by a voltage drop caused by the lightning strike and which resulted in oscillations and discrepancies between the on-shore control systems and individual wind turbines.

The subsequent analysis revealed that there had been performance issues with voltage control when the plant operated at full capacity of $1200 \mathrm{MW}$, but they did not cause deloading and the issue was not communicated to the ESO. Indeed, a similar incident of oscillations happened about 10 mins before the outage, but again at that time the oscillations were well damped and did not cause deloading. A software update to mitigate the problem had been scheduled for 13 August but, because of the outage, it was brought forward to 10 August. 


\subsubsection{Loss of 244 MW steam turbine at Little Barford combined-cycle gas turbine power station}

One second after the lightning strike, the steam turbine at Little Barford tripped because of a discrepancy in the three independent speed sensors on the turbine. This discrepancy exceeded the tolerance of the control system, causing the generator to shut down automatically. The root cause of the discrepancy in the speed sensors has not been established.

\subsubsection{MW DG losses on Loss of Mains protection}

The lightning strike caused sudden voltage changes which were interpreted as islanding by Vector Shift protection. A fast drop in frequency exceeding $0.125 \mathrm{~Hz} / \mathrm{s}$, following the loss of two power plants, was also interpreted as islanding by RoCoF protection on some of DG units. The total infeed loss on Loss of Mains protection was approximately $500 \mathrm{MW}$.

\subsubsection{Frequency response}

A rapid drop of frequency activated the primary frequency response which delivered $83 \%$ of 1022 MW reserve held, i.e. about 850 MW (Ofgem, 2020). The fall in frequency also reduced the demand by about $630 \mathrm{MW}^{8}$. Adding the two components shows that the total infeed loss of $1480 \mathrm{MW}$ was entirely covered by the combination of frequency response and demand reduction so that the frequency stabilised at $49.1 \mathrm{~Hz}$ at $16: 52: 5$, and then started to recover see Figure 3. Hence, if it was not for further infeed losses, the system could have possibly withstood even such a severe disturbance.

\footnotetext{
${ }^{8}$ Many loads consist of rotating machinery so their demand depends on frequency. (NGESO, 2019a) states "For $29 \mathrm{GW}$ demand, this effect is approximately $350 \mathrm{MW}$ at $49.5 \mathrm{~Hz}$ ". Extrapolating to the actual frequency drop of $49.1 \mathrm{~Hz}$ gives $630 \mathrm{MW}$.
} 


\subsection{Second stage: next 11 seconds}

Further infeed losses caused the frequency to fall again-see Figure 3.

\subsubsection{Loss of first (210 MW) gas turbine at Little Barford}

When the steam turbine tripped in the first stage of the event, steam pressure started to rise, and the normal course of action would have been to bypass steam directly into the condenser. For reasons presently unknown, this did not work, and steam pressure continued to rise until after approximately 1 minute the first (GT1a) gas turbine tripped.

\subsubsection{MW DG loss when frequency dropped below $49 \mathrm{~Hz}$}

Distribution Code approved in August 2010 stipulated that under-frequency protection setting of DG should be equal to $47 \mathrm{~Hz}$ for DG with capacities larger than $5 \mathrm{MW}$. However, as probably protection settings on some generators were not changed in line with the new regulations, they tripped when frequency reached the old setting of $49 \mathrm{~Hz}$.

Another possible reason for the embedded generation losses may have been due to internal control systems. Some power electronics interfaced generators may have settings within their internal systems which have been configured by the manufacturer, and as a result, are hidden from the DNO or generators themselves (Ofgem, 2020).

\subsection{Third stage: load shedding and restoration of $50 \mathrm{~Hz}$}

When frequency dropped to $48.8 \mathrm{~Hz}$, automatic load shedding (called LFDD in GB) was activated, and frequency started to recover quickly - see Figure 3. In total 931 MW of demand was disconnected in England and Wales ${ }^{9}$, affecting about 1.1 million people. The power

\footnotetext{
${ }^{9}$ SP Distribution disconnected $22 \mathrm{MW}$ in Scotland, due to incorrect settings in LFDD equipment, and reconnected the customers without informing the ESO.
} 
balance deteriorated again when the second gas turbine unit (GT1b) was tripped manually after 30 seconds by staff at Little Barford power plant due to continuing build-up of steam pressure. Hence further $187 \mathrm{MW}$ was lost, but it did not affect the events materially. After a small dip due to the loss GT1b, frequency continued to recover as the control room instructed generators to increase generation and stabilise the system. In total, about $1240 \mathrm{MW}$ of additional power was delivered and $50 \mathrm{~Hz}$ was restored nearly 5 minutes after the lightning strike. The ESO started to instruct DNOs to restore supply to their customers, and full supply was restored within about 40 minutes.

\subsubsection{Performance of load shedding}

The first stage of LFDD should have resulted in disconnecting the equivalent of $5 \%$ of winter peak demand. However, DNOs disconnected only $3.2 \%$ of the actual demand (ESO, 2019). This has not adversely affected the frequency recovery; however, it is a cause of concern.

There are several explanations provided for the lower levels of demand disconnection. The principal cause appears to be the technical specification of some LFDD relays which prevented them from activating. These relays would have activated if the frequency had dropped marginally lower and the Grid Code permits this margin of error. Another cause may have been the inadvertent disconnection by LFDD of significant volumes of DG which lowered the net demand reduction. Currently, it is not possible to discriminate between sites affected by load shedding on the same feeder - both demand and generation is disconnected. As more and more generation is connected at the distribution level, the current practice of non-discriminative load shedding is becoming increasingly unsustainable.

\subsection{Impact on other critical infrastructures}

The main direct consequence of the event was that about 1.1 million customers lost supply for up to 40 minutes. However, it was the serious impact of the disturbance on other infrastructures 
and services, rather than the power loss itself, which made the outage headline news.

\subsubsection{Rail}

While power supply to the rail network was largely not affected by the load shedding ${ }^{10}$, a certain class of trains (Desiro City Class 700 and 717 manufactured by Siemens and operated by Govia Thameslink Railway (GTR)) suffered a protective shutdown when frequency reached $49 \mathrm{~Hz}$. GTR maintains that the technical specifications for the trains stipulated that they should have operated for a short time when supply frequency drops down to $48.5 \mathrm{~Hz}$. Still, the subsequent investigation discovered that it was not followed. To make things worse, out of 60 trains affected only about half could be restarted by the drivers. The remaining half, which had new software installed, had to wait for a technician to arrive with a laptop to restart it manually, which introduced significant delays.

The impact on the rail network was severe: there were 23 train evacuations, and thousands of passengers had their journeys delayed with 371 trains cancelled, 220 part cancelled, and 873 trains delayed. London St Pancras and King's Cross stations had to close for several hours due to overcrowding, and London Euston went exit only for some time. It all happened on Friday around $5 \mathrm{pm}$, i.e. at the worst possible time, and it was this rail chaos that was the primary source of public anger and made news headlines.

\subsubsection{Other infrastructures}

Two hospitals were disconnected by LFDD, and further two were disconnected by their internal safety systems (E3C, 2020). All hospitals switched to back-up supplies to ensure continued operation.

Two water treatment works were disconnected; one through the LFDD scheme and one as a

\footnotetext{
${ }^{10}$ with exception of one local railway line and three Transport for London stations
} 
result of the company's internal safety systems (E3C, 2020). Additionally, one water company reported a reduction in water pressure to 3000 customers due to booster water pumping stations failing to switch to back-up power automatically. This was reduced to about180 customers within 30 minutes.

Two airports were impacted following the power disruptions (E3C, 2020). Newcastle airport was disconnected from the electricity network following the LFDD and switched to back-up generation. The second airport was not impacted by the LFDD but switched to back-up power supplies. A fault with the on-site internal network disrupted some services for $\sim 50$ minutes.

\subsubsection{Other Energy and the wider industry}

An oil refinery and chemicals manufacturing plant were disconnected due to internal safety systems detecting the drop-in frequency (E3C, 2020). To protect the assets, both plants safely shutdown operations; it took several weeks for the refinery to resume full operations.

\section{WHAT DOES THE OUTAGE TELL US ABOUT THE CURRENT STATE OF DECARBONISED POWER SYSTEMS?}

On the face of it, the power system itself responded exactly how it was designed to do. The system is designed to withstand an (N-1) event, but the outage was caused by simultaneous failures of two power stations, i.e. (N-2) event. Hence the infeed loss was higher than the Secured Event, and the frequency dropped to $48.8 \mathrm{~Hz}$, which triggered load shedding (LFDD). Despite the amount of load shed being less than assumed, and further infeed losses, the frequency was returned to $50 \mathrm{~Hz}$ in nearly 5 mins and power supplies were restored within 40 mins. Consequently, Ofgem basically gave the ESO a clean bill of health. Ofgem was less happy with the owners of the two power stations which failed and two DNOs who were in a 
technical breach of their requirements ${ }^{11}$. They were not fined but agreed to pay "voluntary" payments: Hornsea1 Ltd and RWE Generation UK plc paid $£ 4.5 \mathrm{M}$ each while the two DNOs paid $£ 1.5 \mathrm{M}$ each.

Should we then be happy about the state of the GB power system? The answer is: not really. The blackout has uncovered important fault lines which may significantly affect the reliability of the system in the near future and also affect the connected infrastructures.

\subsection{Does $(\mathrm{N}-1)$ reliability rule need to be reviewed?}

While the system reacted according to the book, i.e. SQSS, the question is if the book is still adequate to the needs. This was recognised by (Ofgem, 2020) which recommended examining if SQSS is fit for purpose with respect to the impact of DG, requirements for holding reserve, response and system inertia. In particular, they recommend assessing whether it is appropriate to provide flexibility in requirements for securing risks events with a very low likelihood, and costs and benefits of requiring availability of additional reserves to secure against the risk of simultaneous loss events. In this section we will look in particular at the latter point, i.e. we will examine whether or not the well-established and universally accepted (N-1) reliability rule should be revised. As the last serious (N-2) incident in GB happened in May 2008 (Bialek, 2020), perhaps the (N-1) principle still holds as one outage per 11 years is quite reasonable from the reliability point of view?

To answer that question, it is important to realise that the (N-1) standard is a common-sense engineering principle that was accepted decades ago when the power supply industry had the following main technical characteristics:

\footnotetext{
${ }^{11}$ Eastern Power Networks and South Eastern Power Networks reconnected demand without instruction from the ESO.
} 
- The main sources of generation were synchronous generators that provided inertia necessary to contain any frequency excursions.

- Power stations were almost exclusively connected to the transmission system so all generation was directly controllable by SO.

- Power stations were also fully dispatchable as they were fed by fossil fuels, water or nuclear energy.

- Distribution networks were passive and did not contain much DG

- SO had detailed models of all main power system elements: generators, their control systems, the transmission system ${ }^{12}$.

Consequently, SO was like an omnipresent and omnipotent god who saw everything and could do almost anything. Of course, the system did evolve, but rather slowly, so SO had time to commission all new equipment properly and consider any interactions. As SO knew the system and its elements very well, it could predict (and eliminate) any common modes of failure and reasonably expect that the probability of two large power stations failing simultaneously and independently was very low.

However, over the past ten years or so the situation has changed quite dramatically in GB and many other countries due to the decarbonisation drive. The main changes were the following:

- There has been a continuous and accelerating replacement of traditional fossil fuel generation by wind and solar. In 2018 renewable generation provided one-third of GB electricity (DBEIS, 2019). Not only wind and solar are not dispatchable but also they do not provide inertia as they are connected to the system by converters.

\footnotetext{
12 Admittedly, load modelling has been a perennial problem.
} 
- In GB, DG is not not visible to the $\mathrm{ESO}^{13}$, which means that effectively DG is treated as negative demand by the ESO. This approach is increasingly non-viable as the amount of DG capacity has increased to reach about 1/3 of the total GB generation capacity in 2019 (DUKES, 2019). The amount of DG that tripped on 9 August was of a similar range as the transmission-connected generation, by some estimates even higher. As (Ofgem, 2020) states: "The event showed that while each distributed generator that de-loaded or tripped may have been small, large volumes of embedded generation behaving in unison can have major impacts on the system. Understanding the behaviour of these generators is critically important for managing the risks to consumers of demand disconnection in a cost-effective manner, and this requires detailed knowledge of their operation and design".

- In addition to wind and solar generation, a significant amount of batteries, active demand and generally smart grids technologies have been added on to the system over the last 10 years. Those converter-connected resources are often equipped with proprietary control systems, models of which are not known to SO, and which means that SO cannot model accurately the system response to disturbances. This was exemplified on 9 August when proprietary voltage control systems of Hornsea wind farm malfunctioned. There can be some possible unstable interactions between control systems which SO is not aware of. To put in simple terms, it means that a lot of new gear and controls were added to the system in a very short time and not all of it was adequately stress-tested and their interactions considered. Indeed, Hornsea 1 offshore power station was progressing through the Grid Code compliance process at the time.

- The ESO has a sophisticated system for contracting frequency response from a variety of providers but there are questions about their compliance. On 9 August, the primary

\footnotetext{
${ }^{13}$ In other countries System Operators have a much better visibility of DG
} 
response providers under-delivered by $17 \%$ and secondary response providers underdelivered by $14 \%$ (Ofgem, 2020). Mandatory response providers and commercial Fast Frequency Response providers of dynamic primary response (required to provide a continuous, proportional response to the change in frequency) performed particularly poorly, under-delivering by approximately $25 \%$ respectively. The reason for the underdelivery is again the fast pace of change. In the old days, when frequency response was delivered only by transmission-connected traditional plants, it was relatively easy to check their compliance with the Grid Code to make sure that they deliver when needed. Now, with a large number of often small providers, checking their compliance is getting increasingly difficult.

- We can only expect that the rate of power system changes will accelerate given the increasingly aggressive decarbonisation targets around the world. For example, the UK has the goal of net-zero greenhouse gas emissions by 2050 .

To summarise the issues outlined above, in the "good old days" SO had to deal with "known unknowns" - they knew the system, they knew what could fail and how. Now we have a lot of "unknown unknowns", i.e. hidden common modes of failures SO does not know about, as demonstrated by the outage on $9^{\text {th }}$ August. Hence it is no longer reasonable to assume that failures of power stations are independent events and therefore $(\mathrm{N}-2)$ event cannot be dismissed as a very rare event. This would suggest that we have two options discussed below.

\subsubsection{Option 1: Business As Usual}

The first option would be to try to maintain the old world with an omnipresent and omnipotent System Operator. That would require System Operator to have full visibility of all generation in the system, both transmission- and distribution-connected. This could be imagined as a hierarchical structure, with SO managing the transmission level and Distribution System Operators (DSOs) taking over some functions of SO and managing the distribution level. 
However if the current trend continues, and all the signs are that it will and may even accelerate to achieve the goal of net-zero carbon economy by 2050, the number of small distributed generators is expected to increase making gathering information and controlling increasingly difficult. Modelling all generators, their controls, distributed storage, active demand and other smart grid technologies would be increasingly difficult even if we assume that it would be possible to force all the companies to disclose how their (often proprietary) controls work. We should strive to achieve as much information as reasonably possible but acknowledge that achieving full observability and controllability is probably a thing of the past.

\subsubsection{Option 2: Provide additional security margin}

The second option is to acknowledge that it is impossible to fully monitor, model and control the whole system at both transmission and distribution levels. That means acknowledging that the probability of common hidden modes of failures has increased and modify security standards to reflect it. While adopting (N-2) criterion might be prohibitively expensive, it might be prudent to consider strengthening the $(\mathrm{N}-1)$ rule by providing extra say $10 \%$ security margin and let us attempt a cost-benefit analysis of that. Our estimates of costs and benefits will be quite approximate and should be treated as order-of-magnitude comparisons.

There are two main components of costs to maintain $(\mathrm{N}-1)$ reliability: frequency reserve and keeping RoCoF below the limit (currently $0.125 \mathrm{~Hz} / \mathrm{s}$ ). NGESO provides regular reports on the cost of ancillary services and here we will use the March 2020 report (NGESO, 2020b). Frequency reserve is considered under the heading Response and in 2019/20 the cost was about $£ 152$ million but unfortunately there is no breakdown of the total into the primary, secondary and high frequency response ${ }^{14}$. Volume-wise, the three components take about one-third each.

\footnotetext{
${ }^{14}$ High frequency response covers actions to reduce frequency when it is going above the statutory limits.
} 
For our approximate analysis let us assume that the costs are also divided one-third each giving a total of about $£ 100$ million for the primary and secondary frequency reserve.

The cost of maintaining the RoCoF limit in $2019 / 20$ was quite high: $£ 210$ million, i.e. more than double of the primary and secondary frequency control. Hence the total cost of $(\mathrm{N}-1)$ security, consisting of the cost of providing frequency reserve and managing RoCoF, was about $£ 310$ million.

To estimate the cost of increasing the security margin we would ideally need the price-quantity characteristics of providing frequency reserve and ROCOF. Unfortunately there is no such information in NGESO reports. Comparing data from different months did not provide any consistent information, probably because conditions and providers changed from month to month. Hence let us undertake an approximate analysis by assuming a general shape of the characteristics.

Assuming that the cost characteristics are linear, $10 \%$ increase in the reserve would cost extra $£ 31$ million/year. However it is likely that the cost-quantity characteristic is non-linear with an increasing slope. Then assuming a quadratic characteristic, $10 \%$ increase in reserve would mean doubling the extra cost to about $£ 62$ million year. Hence we have arrived at a rough estimate of the cost increase to be between $£ 31$ and $£ 62$ million/year.

Now let us consider the benefits of an increased reserve by considering the direct cost of the 9 August outage. It is notoriously difficult to estimate the Value of Lost Load (VOLL) so here let us assume a range $£ 10,000-30,000 / \mathrm{MWh}$ (DECC, 2014). The outage lasted about 40 mins with $931 \mathrm{MW}$ disconnected at the peak giving about $620 \mathrm{MWh}$ of energy lost and therefore the cost in the range $£ 6-18$ million. Incidentally, the outage cost calculated using the often-used VOLL $=£ 17,000 / \mathrm{MWh}$ is exactly equal to the "voluntary" payments of energy companies (£10.5 million) - we do not know whether or not it was accidental. 
One should consider also the cost of failing trains as it was an unexpected cost and therefore not covered by VOLL. UK rail regulations stipulate that a passenger is allowed to the full refund of the ticket if a train is delayed more than 1 hour. The outage happened at evening peak (around 5m on Friday) and most affected were rail services around London. According to (DfT, 2019), two-third of all rail journeys in the UK start or terminate in London, with about 750,000 passengers arriving on average by 9 am and about 170,000 passengers arriving after 5 pm. Of course not all the passengers who arrive by 9 am leave after 5 pm but it is reasonable to expect that a majority will do so for the following reasons: (a) tickets for journeys which finish by 9 am are very expensive with no discounts available so usually a majority of people arriving by 9 am are commuters and business travellers who leave in the evening; (ii) tourists usually arrive after 9 am as they have to travel from other locations. Also, as the disruption happened on Friday evening, it was not only the day commuters, but also week commuters and Londoners going for a weekend retreat, who would leave in the evening. Hence, for our approximate analysis, let us assume that the down factors counterbalance the up factors so the total number of passengers affected was about 920,000. According to (ORR, 2020), the average revenue per rail passenger in 2019 in the UK was $£ 5.59$. Multiplying the number of passengers affected $(920,000)$ by $£ 5.59$ gives the total cost of about $£ 5$ million. This is likely to be a substantial underestimate, as generally tickets from/to London are more expensive than in the rest of the country and the peak-time tickets are especially expensive with no discounts for forward booking. So on the one hand we may have overestimated the number of people affected, but on the other hand, we have underestimated the average ticket price. Hence the error bounds for the cost estimate are quite large so assuming $50 \%$ error, the direct cost of tickets was in the range $£ 3-8$ million.

Adding the outage cost from VOLL (£6-18 million) gives the total direct cost of the outage in the range of $£ 9-26$ million. This estimate does not include other knock-on costs discussed in 
Section 3.4, especially the oil refinery and chemicals manufacturing plants being disconnected for several weeks and therefore likely suffering multi-million losses. It also does not include the knock-on financial effects of train delays or compensation for pain and misery of the travellers. Nevertheless, a comparison with the cost of providing extra $10 \%$ of frequency reserve (£31-£62 million/year) leads to a conclusion that the cost of extra security is likely to be higher than the benefit, even assuming that outages happen every year. Hence, the conclusion would appear to be that, at least in GB, the approximate cost-benefit analysis does not currently support increasing the (N-1) security margin even by as little as $10 \%$.

\subsubsection{How to reduce the cost of providing security?}

It should be emphasised that the above conclusion was based on the static and backwardslooking analysis. There are multiple factors suggesting that the balance of costs and benefits will change in the future. As discussed earlier, it is expected that the frequency of outages may increase due to fast changes happening to the electricity supply industry. RoCoF costs are likely to go significantly down when the current RoCoF limit is increased from $0.125 \mathrm{~Hz} / \mathrm{s}$ to $1 \mathrm{~Hz} / \mathrm{s}$. VOLL is expected to increase due to increased electrification of transport and heating necessary to reach the UK's net-zero emissions target. And finally and most importantly, the cost of providing the security margin is expected to come down due to introduction of innovative frequency controls that use non-traditional providers. As shown in the previous section, maintaining the RoCoF limit in GB is quite expensive ( $£ 210$ million/year) as it is usually done by reducing the imports via DC interconnectors and dispatching instead expensive traditional generation. Wider use of the innovative frequency controls discussed below should significantly reduce the costs.

There are many trials around the world of innovative frequency controls, supplementing the traditional frequency control provided by generators. They essentially consist of inserting energy from a converter-connected energy source (e.g. wind turbine, battery, HVDC 
interconnector, PV not operating at full capacity, supercapacitor) in response to a frequency drop. An alternative to increasing generation is to reduce temporarily demand using devices such as fridges, air-conditioners, heaters etc. and in such a way that the service the devices provide is not affected (Trovato, 2018). The converters can modulate the energy inserted (or withdrawn) according to a pre-specified characteristic. When the amount of energy inserted (or demand reduced) is proportional to the time derivative of frequency $\mathrm{df} / \mathrm{dt}$, the control emulates the inertial effect and therefore is often referred to as the "synthetic (or virtual) inertia". It will have the effect of reducing the initial RoCoF and frequency nadir. On the other hand, if the energy inserted is proportional to the frequency deviation $\Delta \mathrm{f}$, it emulates the droop control of traditional turbines so it reduces the steady-state frequency drop (see Figure 1). A dynamic combination of the inertial $(\mathrm{df} / \mathrm{dt})$ and droop $(\Delta \mathrm{f})$ control can reduce both RoCoF and steadystate frequency drop while eliminating the nadir and minimising the control effort (Mallada, 2016).

It should be emphasised that the "virtual inertia" is not a perfect replacement for the physical inertia. While physical inertia releases kinetic energy automatically, according to the laws of physics, the release of energy by "virtual inertia" suffers from inevitable delays because the control system has to detect and process the frequency signal before reacting to it. It is important to appreciate that frequency cannot be measured directly but only deduced from measurements of AC voltage or current. Mathematically, frequency $\mathrm{f}$ is the derivative of the measured voltage angle. Hence, $\mathrm{df} / \mathrm{dt}$ is the second derivative of the underlying physical quantity which one can measure and therefore it is a very noisy signal.

Measuring df/dt takes 2-3 electrical cycles which for $50 \mathrm{~Hz}$ system means 40-60 ms. The signal is quite noisy so it has to be processed, which takes about $20 \mathrm{~ms}$. Then the energy source behind the converter has to be activated and the delay depends on the technology used. It takes at least $4 \mathrm{~ms}$ for a flywheel, 10-20 ms for batteries or supercapacitors, 40-500 ms for a wind turbine, 
100-200 ms for solar PV and 50-500 ms for HVDC (GE, 2017). Hence, the total delay between the disturbance and activation of "virtual inertia" can be somewhere between $0.1 \mathrm{~s}$ and $0.5 \mathrm{~s}$. Currently such delay is acceptable for most systems as they still have a considerable amount of physical inertia installed which limits the initial RoCoF. However, in some systems with a high penetration of wind/solar (like e.g. South Australia), the RoCoF following a large infeed loss may reach about $5 \mathrm{~Hz} / \mathrm{s}$, so $0.1-0.5 \mathrm{~s}$ delay would mean a drop of frequency of $0.5-2.5 \mathrm{~Hz}$ before "virtual inertia" is activated. This may have serious consequences for frequency stability as the delayed release of "virtual inertia" will result in a deep frequency nadir which may inadvertently activate under-frequency load shedding. Hence "virtual inertia" cannot replace physical inertia completely and maintaining some physical inertia on the system may be necessary. It could be done by e.g. keeping the generators of decommissioned traditional plants connected to the system so that they still provide physical inertia ${ }^{15}$. Some countries consider introducing "inertia markets" that would provide a level-playing field for different providers (Poolla, 2020). However currently utilities tend to prefer including the provision of inertia and innovative frequency controls in the existing ancillary services markets (AEMC, 2018), (NGESO, 2020a). As more services are added, and more providers enter, the costs are expected to come down.

It should be noted that there is a new technology of so-called grid-forming converters which do not rely on the physical inertia of rotating masses to keep power system balance and therefore do not require measuring frequency with associated delays (Paolone, 2020). Wider application of those new techniques may lead in future to inertia-less systems. This however would require a fundamental change of paradigm of the current power system operation and

\footnotetext{
${ }^{15}$ Such generators are referred to as synchronous condensers as traditionally they have been used for reactive power compensation, i.e. voltage control.
} 
control which we will not discuss here.

An alternative to providing system security by maintaining a passive (and expensive) security reserve is to rely on emergency controls, referred to as Special Protection Systems (SPS) or Remedial Action Schemes (RAS), that react to specific contingencies (ENTSOE, 2012). There are many RAS proposed in the literature and it is not the aim of this paper to discuss them. One example is early activation of under-frequency load shedding based on the RoCoF signal, rather than frequency drop (Banijamali, 2019). As the size of the infeed loss $\Delta \mathrm{P}$ can be estimated from RoCoF (knowing system inertia $\mathrm{H}$ - see equation (1)), load shedding can be made more accurate and smaller.

Innovative frequency controls have been around for some time however we believe that a major impediment to their wider application is not technical but institutional. SOs tend to be conservative and prefer maintaining a large security margin and physical inertia which, while more expensive, is regarded to be more reliable than controls which might malfunction or function when not needed. Nevertheless, the increased probability of outages, combined with a high cost of increasing the security margin and maintaining physical inertia, could force SOs to consider innovative frequency controls more widely.

\subsection{Load shedding}

The outage has demonstrated that the effectiveness of load shedding in reducing the overall demand could be much lower than expected due to indiscriminate shedding of all customers on the disconnected feeders, including DG. As the amount of DG is very likely to increase in the coming years, we believe it is highly desirable to consider how to make load shedding more flexible and selective. With rapid advances in telecommunication, it should be possible to assess in real-time the actual loading on individual feeders, so that load shedding has the maximum possible effect. Another option could be to consider if it would be feasible and cost- 
effective to implement load shedding at $11 \mathrm{kV}$ level, rather than $33 \mathrm{kV}$ as presently, hence allowing more selective operation (Bell, 2019).

\subsection{Interactions between critical infrastructures}

The power network is only one part of a highly complex system containing other infrastructures, such as transport, water, communications and other, which are interconnected physically and through a flow of data and information. Interactions between those subsystems are still poorly understood, and there is a significant risk that if the current compartmentalised approach to their governance and operation is not changed, we may see more unexpected consequences of disturbances propagating across the whole system, as indeed happened on 9 August. Hence there is an urgent need to understand better all the interactions and change the technical governance of these complex systems to be more flexible and embracing all the system participants. Compliance with the regulations must be enforced to make sure that other infrastructures can survive power disturbances and accompanying large frequency deviations.

\section{CONCLUSIONS AND POLICY IMPLICATIONS}

Over the last 10-15 years, power systems in many countries have changed quite rapidly and significantly with renewables replacing traditional fossil-fuel generation, large penetration of DG in distribution networks, and increasing deployment of energy storage, active demand and generally smart grids technologies. The 9 August 2019 GB power outage was a unique stress test exposing the fault lines brought about by the rapid changes. On the face of it, the power system itself responded exactly how it was designed to do. A lightning strike caused two power stations to trip, so it was (N-2) event compounded by a resulting loss of DG. Consequently, the frequency was falling rapidly triggering under-frequency load shedding which restored power balance. Should we then be happy about the state of the GB power system? The answer is: not really. The blackout has uncovered important problems that may significantly affect the system 
reliability in the near future.

The decarbonisation drive resulted in a significant amount of new equipment and controls added to the system in a very short time. All the new equipment has its own sophisticated control systems with possibly some unknown interactions. Also the system inertia has been significantly reduced. The pace of change is likely to increase due to the adoption of ambitious environmental targets such as net-zero emission by 2050 . Hence it will be increasingly difficult for SO to fully monitor, model and control the whole system and therefore the probability of common hidden modes of failures, as the one exposed by the 9 August outage, will be significantly increased. The business-as-usual option is not feasible so it might be prudent to reconsider the old $(\mathrm{N}-1)$ security standard by providing an additional reserve. While the approximate cost-benefit analysis has shown that increasing the security reserve is currently too expensive, the balance of costs and benefits is likely to change in future. In particular, the costs can be significantly reduced by introducing a range of innovative frequency controls, including the provision of "virtual inertia" and Remedial Action Schemes. However "virtual inertia" suffers from measurement and processing delays so maintaining some amount of physical inertia, e.g. by using synchronous condensers, may be necessary to limit RoCoF. This may change in future when wider application of grid-forming converters may make it possible to achieve inertia-less systems. One of the main impediments to introducing innovative controls is the attitude of SOs who tend to be conservative and prefer maintaining a large security margin and physical inertia which, while more expensive, are regarded to be more reliable than controls which might malfunction or function when not needed.

There were also other issues highlighted by the outage. DG reached such a high penetration level that it cannot be treated any longer as negative demand. Traditional under-frequency load shedding disconnects all customers indiscriminately on the disconnected feeders, including embedded generation and frequency response units. With rapid advances in 
telecommunication, it should be possible to assess in real-time the actual loading on individual feeders so that load shedding has the maximum possible effect and perhaps also implement load shedding at $11 \mathrm{kV}$ level, rather than $33 \mathrm{kV}$, hence allowing more selective operation.

Another important issue is the ability of critical infrastructures and services to ride through power and frequency disturbances. The main adverse effect of the 9 August blackout was not the loss of supply itself, although it affected 1.1 million customers, but a severe disruption to rail service around London due to an unexpected failure of trains when frequency dropped to $49 \mathrm{~Hz}$. The power network is only one part of a highly complex system containing other infrastructures which are interconnected physically and through a flow of data and information. Interactions between those subsystems are still poorly understood, so the current compartmentalised approach to their governance and operation should be changed to avoid more unexpected consequences of spreading disturbances across the whole system.

\section{REFERENCES}

(AEMC, 2018) Australian Energy Market Commission: "FINAL RULE DETERMINATION. National Electricity Amendment (Inertia Ancillary Service Market) Rule 2018, 6 February 2018

(Banijamali , 2019) S. S. Banijamali, T. Amraee: "Semi-Adaptive Setting of Under Frequency Load Shedding Relays Considering Credible Generation Outage Scenarios”, IEEE Trans. Power Delivery, v. 34, June 2019

(Bell, 2019) K. Bell: “What happened on August $9^{\text {th }}$ - the investigations", UK Energy Research Centre

(Bialek, 2020) J. W. Bialek "What does the power outage on 9 August 2019 tell us about GB power system”, EPRG Working Paper, 2020 
(DBEIS, 2019): Department for Business, Energy and Industrial Strategy: "Electricity Supply Emergency Code (ESEC)”, 2019

(DECC, 2014) Department for Energy and Climate Change "Electricity Market Reform. Impact Assessment”, IA No: DECC0151, 4 September 2014

(DfT, 2019) Department for Transport: "Rail Factsheet. December 2019”

(DUKES, 2019): Department for Business, Energy and Industrial Strategy: "Digest of UK Energy Statistics, 2019"

(E3C, 2020): Department for Business, Energy \& Industrial Strategy: “GB Power system disruption - 9 August 2019. Energy Emergencies Executive Committee: Final Report”, 2020

(ENTSOE, 2012) European Network of Transmission System Operators for Electricity (ENTSOE): “Special Protection Schemes”, March 2012

(GE, 2017) GE Energy Consulting: "Final Report: Technology Capabilities for Fast Frequency Response”, March 9, 2017

(Mallada, 2016) E. Mallada: "iDroop: A dynamic droop controller to decouple power grid's steady-state and dynamic performance," 55th IEEE Conference on Decision and Control (CDC), Dec. 2016

(NGESO, 2019a) national gridESO: "Interim Report into the Low Frequency Demand Disconnection (LFDD) following Generator Trips and Frequency Excursion on 9 August 2019”, $16^{\text {th }}$ August 2019.

(NGESO, 2019b) national gridESO: “Technical Report on the events of 9 August 2019”, 6 September 2019.

(NGESO, 2020a) national gridESO: “Operating a Low Inertia System. A System Operability Framework Document” February 2020. 
(NGESO, 2020b) national gridESO: "Monthly Balancing Services. Summary 2019/20. March 2020"' February 2020.

(Ofgem, 2020): “9 August power outage report”, 3 January 2020

(ORR, 2020) Office of Rail and Road: "Passenger Rail Usage. 2019-20 Q4 Statistical Release”, 4 June 2020

(Paolone, 2020) M. Paolone, T. Gaunt, X. Guillaud, M. Liserre, S. Meliopoulos, A. Monti, T. Van Cutsem, V. Vittal, C. Vournas "Fundamentals of Power Systems Modelling in the Presence of Converter-Interfaced Generation” Power System Computation Conference, 2020.

(Poolla, 2020) B. K. Poolla, S. Bologniani, N. Li, F. Doerfler: “A Market Mechanism for Virtual Inertia” IEEE Trans. Smart Grid, v. 11, 2020

(SQSS, 2019): "National Electricity Transmission System Security and Quality of Supply Standard", 2019

(Teng, 2015) Teng, F., Aunedi, M, Pudjanto, D, Strbac, G: "Benefits of Demand Side Response in Providing Frequency Response Service in the Future GB Power System”, Frontiers in Energy Research; v. 3; 2015.

(Trovato, 2018): V. Trovato, F. Teng, G. Strbac: "Role and Benefits of Flexible Thermostatically Controlled Loads in Future Low-Carbon Systems”, IEEE Trans. Smart Grids, v. 9 , Sept. 2018 